\title{
Early Childhood Caries: Policy and Prevention
}

\author{
Francisco Ramos-Gomez
}

\begin{abstract}
Background: Early childhood caries (ECC) is the most common chronic disease in children, aged 2-5 years, and is considered a priority action area by the World Dental Federation (FDI). ${ }^{1,2}$ The prevalence of ECC in children in India is extremely high and calls for immediate action. ${ }^{3}$ There is an urgent need for evidence-based oral health care and for the development of policies that support education, prevention, and healthy behaviors, specifically a national policy that recommends every child be seen by a dentist before the age of 1 year. Oral health is a matter of quality of life, overall health, social justice, and human rights.

Materials and methods: There is a need to advocate for and promote a mandatory age-one visit to assess a child's risk level for ECC and establish a primary prevention plan. Effective oral health assessments for infants include six steps: caries risk assessment; knee-to-knee positioning of the child; toothbrush prophylaxis; a clinical examination; fluoride varnish treatment; and anticipatory guidance, counseling, and self-management goals.

Recommendations: In order to increase oral health literacy and improve the oral health of children, certain recommendations should be prioritized and adhered to. These include the inclusion of risk assessment, anticipatory guidance, and self-management goals, an emphasis on ECC and oral health education, prioritizing primary prevention of ECC through healthy behaviors and the promotion of a mandatory age-one visit policy, interprofessional collaboration, and continued research of ECC inequalities and how to address them.
\end{abstract}

Keywords: Dental caries, Infant care, Oral health, Pediatric dentistry, Perinatal care, Prevention and control, Risk assessment, Socioeconomic factors. Journal of South Asian Association of Pediatric Dentistry (2020): 10.5005/jp-journals-10077-3040

\section{INTRODUCTION}

During my exciting and eye-opening trip to India, I had the amazing opportunity to visit five different cities and interact with my colleagues with presentations over the course of 10 days. Through this experience, I encountered first-hand understanding that the prevalence of early childhood caries (ECC) in India's children is high, and there is a critical need to address this issue through evidencebased oral health care and policies that support education, prevention, and healthy behaviors. ${ }^{3}$ I came away convinced of the urgency of India's battle against ECC, as a matter of not only oral health but also quality of life, overall health, social justice, and human rights.

\section{BACKGROUND OF ECC}

The World Dental Federation (FDI) defines ECC as "a very common bacterial infection and multifactorial disease characterized by marked decay of the teeth of children 6 years of age or younger..11,2 As the most frequently occurring chronic disease in children aged $2-5$, ECC has become more and more widespread around the world and is considered a priority action area by the FDI. ${ }^{1}$ Even though it can be prevented, many cases of ECC remain untreated, and over 600 million children are affected by dental caries. ${ }^{2,4}$

Many factors contribute to a child's risk level for ECC. Oral health begins when a child is in utero and is affected by the oral health of the mother. If the mother's oral health is poor, it could interfere with the development of the child's enamel, making them more susceptible to ECC. ${ }^{1,5}$ Once the child is born, caries-producing bacteria can be transmitted to children from the caregiver. Studies show a link between a caregiver's bacteria levels and the child's level of risk for caries, ${ }^{6}$ emphasizing the importance of perinatal oral care for expectant mothers as a contributing factor of the child's oral health. ${ }^{7}$ Like other non-communicable diseases, ECC is connected to sugar intake, which prolongs acid production from tooth adherent bacteria, affecting the makeup of the oral microbiota and biofilm
UCLA School of Dentistry, Section of Pediatric Dentistry, University of California, Los Angeles, California, USA

Corresponding Author: Francisco Ramos-Gomez, UCLA School of Dentistry, Section of Pediatric Dentistry, University of California, Los Angeles, California, USA, Phone: +1 310825 9460, e-mail: frg@ dentistry.ucla.edu

How to cite this article: Ramos-Gomez F. Early Childhood Caries: Policy and Prevention. J South Asian Assoc Pediatr Dent 2020;3(1):3-6. Source of support: Nil

Conflict of interest: None

$\mathrm{pH}$ and ultimately demineralizing the teeth and leading to defects in the development of the enamel. ${ }^{2}$

Because ECC on primary teeth has negative effects on the overall health of the child, it is crucial that primary teeth are kept healthy. ${ }^{2}$ Untreated caries cause pain and infection and can deter activities like eating, speaking, and learning, consequently harming cognitive development, school readiness, and selfesteem. ${ }^{1}$ Caries in a child do not disappear when that child becomes an adult; on the contrary, the presence of ECC in a child's primary teeth increases the likelihood of decay in the child's mixed and permanent dentition. ${ }^{1}$

In the United States and many other countries, the highest rate of caries exists in children from low-income and minority backgrounds. ${ }^{8}$ Unfortunately, efforts to address ECC have not always been successful, possibly due to the fact that they have not considered the social determinants that impact families and influence behavioral issues. ${ }^{9}$ Because treatment of ECC is both expensive and connected to health risks, and because of the high rate of recurrence after such treatments, ${ }^{10,11}$ the emphasis has shifted towards primary prevention of ECC. ${ }^{10-12}$

The Centers for Disease Control and Prevention defines the three levels of prevention as follows: ${ }^{13}$ 
- Primary prevention refers to prevention that occurs before the presence of a disease or condition. This is done through avoiding substances and behaviors known to increase risk and taking precautionary steps to prevent the onset of disease. ${ }^{13}$

- Secondary prevention includes identifying the disease before symptoms begin to show through regular exams and/or testing. ${ }^{13}$

- Tertiary prevention refers to how a disease is managed after diagnosis and the steps that are taken to hinder or stop disease progression. ${ }^{13}$

These three levels of prevention can be applied more specifically to ECC:

- Primary prevention of ECC: Stop caries from happening in the first place through anticipatory guidance, the early establishment of a dental home, frequent oral health visits starting from an early age, and good oral health habits with the application of fluoride varnish at the age one visit.

- Secondary prevention of ECC: Detect, track, and treat noncavitated, carious lesions early on when they are still reversible. Prevent and treat them from progressing further through fluoride application, combination therapy approach, selfmanagement goals, etc.

- Tertiary prevention of ECC: Repair and restore cavitated, carious lesions with SDF, glass ionomers, and other pertinent restorative dental materials.

Of the three levels of prevention of ECC, primary is most important because it addresses the disease before it begins, minimizing or eliminating the need for secondary and tertiary prevention later on. ${ }^{13}$

\section{Background of the Age 1 Visit}

In 2014, the FDI adopted a new policy for perinatal and infant oral health. ${ }^{1}$ Although the policy did not refer to establishing a dental home, it did stress the importance of parental involvement in a child's oral health and recommended parents begin practising preventive behaviors when the first tooth erupts, or no later than 12 months old. ${ }^{1}$

In their 2019 Bangkok Declaration, the International Association of Paediatric Dentistry (IAPD) recommended that all children receiving care from health professionals and community workers also be given guidance regarding ECC prevention. ${ }^{2}$ Ideally, this preventive guidance would result in a referral to a dentist. ${ }^{2}$ The current stance of the IAPD is that dental visits are recommended upon the eruption of the first tooth or by 1 year so that parents can gain advice and learn about the best care and prevention practices from the dentist. ${ }^{14}$

\section{Six Steps of an Infant Oral Care Visit ${ }^{15}$}

An effective oral health assessment consists of six steps that will make infant oral visits easier for dental care providers.

\section{Caries Risk Assessment}

Begin by performing a caries risk assessment such as the caries management by risk assessment interview form ${ }^{15}$ (see page 8 ). Greet the child and caregiver, explaining what will happen during the visit and answering whatever questions they might have. Offer positive feedback to help the caregiver relax. Gather important information about risk factors and behaviors that can aid the development of an individualized treatment plan based on the child's level of risk. Questions should address the following:
- Child's overall general health;

- Access to fluoridated water or fluoride supplements;

- Toothbrushing routines and the use of fluoridated toothpaste;

- Oral health history of child and parents;

- Last dental visit;

- Known caries in the child, siblings, or parents;

- Bottle-feeding and sippy cup usage, including contents and frequency;

- Diet and snacking habits.

The interview should be conducted respectfully and with cultural sensitivity. The examiner should recognize the knowledge, experiences, values, and views of the caregiver and should listen to any ideas or family perspectives that the caregiver offers. Culturally and linguistically, appropriate tools should be used to discuss oral health.

\section{Proper Positioning of the Child}

Use the knee-to-knee position for children aged 6 months to 3 years (or up to 5 years for children with special needs) during the clinical examination and fluoride application. Children older than 3 years can sit forward on their caregiver's lap or alone in a chair. Work with the caregiver to ensure a smooth transition between the interview and the examination. Before starting, explain what will happen using the Tell, Show, and Do method. Expect that the child might cry, and be aware that this reaction is developmentally appropriate for babies. Knee-to-knee positioning includes:

- The examiner and caregiver face each other, knee to knee.

- The child sits in the caregiver's lap while facing the caregiver.

- With the child's legs wrapped around the caregiver's waist, the caregiver holds the child's hands. The child's body is laid down on the caregiver's lap.

- The child's head is laid in the examiner's lap.

- Still holding the child's hands, the caregiver uses her arms/ elbows to hold the child's legs steady against her waist.

- The examiner positions the child's head and prompts him to open his mouth for the toothbrushing prophylaxis.

\section{Age-appropriate Toothbrush Prophylaxis}

The examiner should retract the child's lips and cheeks throughout the toothbrushing process so that the caregiver can observe the technique and understand the proper way to brush the child's teeth. Use the spongy handle of an age-appropriate-sized toothbrush to prop open the child's mouth. The caregiver should be encouraged to brush both her and her child's teeth with fluoridated toothpaste of at least 1000 ppm.

\section{Clinical Examination of the Oral Cavity and Dentition}

The examiner should count the child's teeth aloud. If necessary, this task can be made into a game by singing songs, engaging the child's attention, or distracting the child with a colorful toy. Reward cooperation and good behavior with praise.

In addition to a soft tissue examination, the examiner should document

- The presence and location of any visible plaque;

- Chalky white spots;

- Brown spots that indicate decay;

- Tooth defects deep pits/fissures and tooth anomalies;

- Any oral or tooth abnormalities; 
- Missing or decayed teeth;

- Existing restorations;

- Untreated caries and/or defective restorations;

- The presence and location of gingivitis and/or any soft tissue abnormalities;

- Occlusal status, malocclusion, or developmental pathology

- Indications of trauma.

If possible, a salivary analysis should also be performed in order to measure Streptococcus mutans, Lactobacilli levels, or the acidogenic potential of biofilm.

All results from the clinical examination should be combined with data from the interview in step 1 in order to determine the child's risk for caries, his or her oral diagnosis, and an individualized plan for treatment.

\section{Fluoride Varnish Treatment}

For children who are moderate to high risk for caries, apply fullmouth topical fluoride varnish and recommend reapplication every 3-6 months. Emphasize the benefits of fluoride varnish and remind the caregiver that the child should not drink for 30 minutes after the treatment so that the fluoride varnish will be effective.

\section{Anticipatory Guidance, Counseling, and Self-management Goals}

Conclude the oral examination by sitting the child back up, praising him or her and offering a reward (a sticker, toy, etc.).

The following areas should be discussed with the parent/ caregiver based on the child's age and risk level:

- Oral health and hygiene: As soon as a child's teeth begin to erupt, parents should brush the teeth twice a day. Parents should begin flossing their child's teeth once the child's teeth are touching. ${ }^{17}$

- Oral development: Discuss teething and what can be expected with the parent.

- Fluoride adequacy: While each child will have unique needs, parents should be informed about the importance of both topical and systemic fluoride exposure for their children's oral health including using fluoridated toothpaste. ${ }^{15}$

- Oral habits: Check oral habits that may affect dentition, including digit sucking, pacifier use, tongue thrusting, and object biting. ${ }^{18}$

- Diet and nutrition: Parents should be taught to limit their child's consumption of fruit juices, clean their child's mouth with a damp cloth after eating, and only let their child go to bed with a bottle that contains water. ${ }^{15}$ The IAPD advises that free sugars (which include added sugars as well as the natural sugars in honey, syrup or fruit juices) be eliminated from the diets of children 2 years of age or younger. ${ }^{2}$

- Injury prevention: Because of the prevalence of head injuries among pediatric emergency department visits, taking precautions to prevent injury is important for both oral and overall health. Strategies for prevention include the use of safety gates, rear-facing car safety seats, and adult supervision. ${ }^{19}$

Also be sure to discuss the results of the child's risk assessment interview and clinical examination with the caregiver, explaining what risk level means to the long-term oral health of the child. Answer the caregiver's questions regarding a treatment plan and offer preventive counseling specific to their child. A mutually agreed upon self-management plan should also be established with self-management goals ${ }^{16}$ such as providing healthy snacks, brushing with fluoride toothpaste, etc. (see page 9).

\section{Policy Recommendations}

\section{Risk Assessment, Anticipatory Guidance and Self-management Goals}

The inclusion of individual risk assessments, anticipatory guidance, and self-management goals during clinical examinations is encouraged during the child's first year as a part of diagnosing and planning treatment. ${ }^{1,2}$ Risk assessments address risk factors (e.g., continual bottle use, diet, medications), protective factors (e.g., use of fluoridated toothpaste, access to fluoridated water). They also address clinical findings such as demineralized enamel, cavitated lesions, etc. ${ }^{16}$

Providers should address current behaviors and counsel parents/caregivers about setting appropriate self-management goals based on the results of the child's caries risk assessment. The goals should be focused on the patient and specific to their individual risk factors. These may include the beliefs and practices of the family regarding diet, bottle-feeding, brushing with fluoridated toothpaste, and the importance of keeping primary teeth healthy.

Anticipatory guidance can come from health professionals or community health workers and should ideally lead to a dental referral for the establishment of a dental home and continuation of the child's oral health care. ${ }^{2}$

\section{Oral Health and ECC Education}

Both the IAPD and the FDI recommend that measures be taken to increase awareness of ECC through the education of parents/ caregivers, dentists, dental hygienists, physicians, nurses, health professionals, and other invested parties. ${ }^{2}$ Both health-care providers and caretakers need to understand that the development of fetal dentition is affected by the mother's oral health and dental care during pregnancy. They should also be made aware of the maternal factors that could harm the child's oral health development, ${ }^{1}$ including high maternal pathogenic bacterial levels, high snacking frequency, and poor maternal oral hygiene. ${ }^{20}$

The IAPD recommends the following four priority areas in the prevention of ECC: ${ }^{18}$

- Raising awareness of ECC among parents, caregivers, dentists, nurses, pediatricians, and other health professionals and invested parties.

- Limiting sugar intake in children's diets and avoiding free sugars for children younger than 2 years.

- Brushing teeth twice per day with an age-appropriate amount of fluoridated toothpaste of at least 1,000 ppm.

- Providing first preventive guidance by the age of 1 year. This guidance should come from a health professional or community health worker and should ideally result in a referral to a dentist for continuing care.

Additionally, dental schools are encouraged to incorporate curriculum on ECC so that evidence- and risk-based prevention strategies are presented with equal weight to traditional surgical methods. ${ }^{2}$ Through an emphasis on increasing oral health literacy, health equity can improve.

\section{Preventive Care through Healthy Behaviors}

The FDI acknowledges that effective early intervention relies on effective prenatal counseling and education as well as the oral 
health care given at the perinatal and infant stages of a child's life. This is the best time for healthy behaviors and habits to be established, which, in turn, will help prevent ECC and keep oral

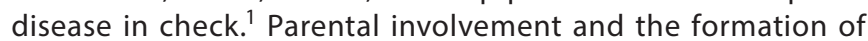
healthy habits related to oral health should be encouraged by health providers across pediatric disciplines upon the eruption of a child's first tooth or no later than 12 months old.

\section{Interprofessional Collaboration}

The FDI states that the future of oral health is linked to an interprofessional and multidisciplinary integration of oral health into primary care. They recommend interprofessional collaboration between medical, dental, and other health-care practitioners, allowing the settings for the prevention and management of ECC to be extended to various primary care locations and administered by qualified pediatric health-care professionals. ${ }^{3}$ Such collaboration contributes to the more effective education of parents and caregivers in a culturally and linguistically appropriate manner, while also allowing important information regarding oral health to be more available to pregnant women, infants, and children, specifically those from vulnerable, high-risk backgrounds.'

Additionally, both the IAPD and the FDI recommend the involvement of multiple parties in order to address the various sides of ECC. Because there are so many factors involved in this disease, collaboration and cooperation between stakeholders is necessary for prevention. ${ }^{2}$ Advocacy for the improvement of quality and availability of care to the underserved through interprofessional education and practice is encouraged, since providing training about oral health to medical and nursing professionals will allow the oral health of more children to be assessed at an earlier age, especially because infants and children more commonly attend early primary care visits rather than dental visits. ${ }^{1}$

\section{Research}

The IAPD recommends the support of research that investigates ECC inequalities, quality of life as connected to oral health, interventions, and health economics. Research in these fields will allow for a better understanding of the benefits of care when offered in an effective and timely manner. ${ }^{2}$

\section{Conclusion}

The effects of ECC on the children of are far-reaching, with negative effects that impact well-being in its entirety. An increase of oral health literacy among the public and of accessible care for the prevention of ECC will rely on interprofessional collaboration and cooperation between medical, dental, and other health-care providers throughout the country. By extending the settings of oral health evaluations beyond dental offices and into primary care locations, more children will receive individual risk assessments by the age of 1 . As a result, more families will receive anticipatory guidance and education about oral health behaviors, and ultimately more cases of ECC will either be prevented or addressed at earlier stages.

I was humbled by the opportunity to visit India and by the expertise and vision of my colleagues and fellow presenters. There is work to be done, but I am convinced that India has the resources needed to combat the prevalence of ECC nationwide, to spread awareness of the vital role oral health plays in a child's overall health and to increase accessibility to care for all of the nation's children.

\section{References}

1. World Dental Federation. Perinatal and Infant Oral Health. https:// www.fdiworlddental.org/resources/policy-statements-andresolutions/perinatal-and-infant-oral-health. Adopted September 2014. Accessed December 4, 2019.

2. Pitts N, Baez R, Diaz-Guallory C, et al. Early Childhood Caries: IAPD Bangkok Declaration. Int J Paediatr Dent 2019;29:384-386.

3. Ganesh A, Muthu MS, Mohan A, et al. Prevalence of early childhood caries in India - A systematic review. Indian J Pediatr 2019;86:276-286. DOI: 10.1007/s12098-018-2793-y.

4. Ramos-Gomez FJ, Crystal YO, Domejean S, et al. Minimal intervention dentistry: part 3. Paediatric dental care--prevention and management protocols using caries risk assessment for infants and young children. Br Dent J 2012;213(10):501-508. DOI: 10.1038/sj.bdj.2012.1040.

5. Ramos-Gomez FJ, Crystal YO, Ng MW, et al. Pediatric dental care: prevention and management protocols based on caries risk assessment. J Calif Dent Assoc 2010;38(10):746-761.

6. Douglass J M, Li Y, Tinanoff N. Association of mutans streptococci between caregivers and their children. Pediatr Dent 2008;30:375-387.

7. Ramos-Gomez F. Bacterial salivary markers' role in ECC risk assessment in infants. J Dent Res 2006;85B:poster 0516.

8. Jackson SL, Vann WFJr, Kotch JB, et al. Impact of poor oral health on children's school attendance and performance. Am J Public Health 2011;101(10):1900-1906. DOI: 10.2105/AJPH.2010.200915.

9. Ramos-Gomez F. Understanding oral health disparities in the context of social justice, health equity, and children's human rights. J Am Dent Assoc 2019;150(11):898-900. DOI: 10.1016/j.adaj.2019.09.004.

10. Sinner B, Beck K, Engelhard K. General anesthetics and the developing brain: An overview. Anesthesia 2014;69(9):1009-1022.

11. Berkowitz RJ, Amante A, Kopycka-Kedzierawski DT, et al. Dental caries recurrence following clinical treatment for severe early childhood caries. Pediatr Dent 2011;33(7):510-514.

12. American Academy of Pediatric Dentistry. Perinatal and infant oral health care. Reference manual 2018-2019. Pediatr Dent 2018; 40(6):216-220.

13. Centers for Disease Control and Prevention. Prevention. Picture of America. Available at https://www.cdc.gov/pictureofamerica/pdfs/ picture_of_america_prevention.pdf. Accessed March 17, 2020.

14. International Association of Paediatric Dentistry. Children 0-2 years of age: Frequently Asked Questions. http://www.iapdworld.org/parents/ super_pages.php?ID=parents1\#6. Accessed December 4, 2019.

15. Ramos-Gomez F. Six Step Protocol for a Successful Infant Oral Care Visit. Pediatric Dentistry Today 2009. 38-40.

16. Ramos-Gomez F, Ng MW. Into the future: keeping healthy teeth caries free: pediatric CAMBRA protocols. J Calif Dent Assoc 2011;39(10): 723-733.

17. American Academy of Pediatrics. Oral Health Risk Assessment Timing and Establishment of the Dental Home. Pediatrics 2013;111(5): 1113-1116.

18. International Association of Paediatric Dentistry and Alliance for a Cavity-Free Future. Action on Early Childhood Caries from multiple stakeholders is needed now in four key areas. Infographic. An output from the IAPD Global Summit on Early Childhood Caries, 2018.

19. Taylor CA, Bell JM, Breiding MJ, et al. Traumatic brain injury-related emergency department visits, hospitalizations, and deaths - United States, 2007 and 2013. MMWR Surveill Summ 2017;66(9):1-16. DOI: 10.15585/mmwr.ss6609a1.

20. Tinanoff N, Kanellis MJ, Vargas CM. Current understanding of the epidemiology mechanisms, and prevention of dental caries in preschool children. Pediatr Dent 2002;24:543-551. 